\title{
THE IMPORTANCE OF THE OHI-S FOR CHILDREN WITH PREMATURE TOOTH LOSS
}

\author{
Radosveta Andreeva ${ }^{1}$, Ani Beltcheva ${ }^{2}$ \\ ${ }^{1}$ Department of Pediatric Dentistry, Faculty of Dental Medicine, \\ Medical University of Varna \\ ${ }^{2}$ Department of Pediatric Dentistry, Faculty of Dental Medicine, \\ Medical University of Plovdiv
}

\begin{abstract}
In spite of all the methods of prevention, there still are untreated or improperly treated caries and it's followed by complications. This often leads to premature loss of teeth.

The premature loss of the deciduous teeth depends on different factors such as oral hygiene, traumas, complicated caries. The aim of this study is to compare the OHI-S between children with premature loss and those without premature loss of deciduous teeth. Subject of the monitoring of the clinical research were 140 children between ages 6 and 9. The clinical group consisted of 90 children with prematurely extracted teeth, divided into three groups. The control group consisted of 50 children with intact denture. The oral hygiene status was examined and registered by the Greene-Vermillion index-simplified. There was a statistically significant difference in the OHI-S between the control group $(1.86 \pm 0.05)$ and the clinical groups $(1.90 \pm 0.08$ and $2.01 \pm 0.05)$. The children with premature extraction of the primary teeth had higher OHI-S compared to the control group (children without premature extraction of teeth). The third group of children, with prematurely extracted temporary teeth and severe orthodontic deformation, have the highest $\mathrm{OHI}-\mathrm{S}$. These results demonstrate the connection between $\mathrm{OHI}$, the orthodontic deformations and the need for prevention.
\end{abstract}

Keywords: premature loss, caries, premature extraction, OHI-S, oral hygiene

\section{INTRODUCTION}

In spite of all the methods of prevention, there are still untreated or improperly treated caries and it's followed by complications. This often leads to premature loss of teeth (11).

The premature loss of the deciduous teeth depends on different factors such as oral hygiene, traumas, complicated caries (5).

Address for correspondence:

Radosveta Andreeva

Faculty of Dental Medicine,

Medical University of Varna

55 Marin Drinov Str.

9002 Varna, Bulgaria

email:doctor_ra@abv.bg

Received: April 12, 2015

Accepted: May 11, 2016
The carious lesion is a disease of the hard dental tissues with a wide prevalence among the young population (1). The premature extraction of the primary teeth is the way of treatment of complicated caries in many countries $(3,4)$. It often leads to a lack of space for the permanent teeth. The poor oral hygiene is one of the main etiological factors for the occurrence of carious lesions, which are the main factor for premature tooth loss (10). There is a close relationship between the oral hygiene, caries activity and the premature tooth loss. According to the literature a significant part of the school children have carious lesions - about 60-90\% - WHO, (2012). A lot of temporary teeth can be preserved from premature extraction if the oral hygiene is adequate. 


\section{AIM}

The aim of this article is to compare the $\mathrm{OHI}$ between children with premature extraction and those without such problems.

\section{MATERIALS AND METHODS}

The subject of monitoring of the clinical research were 140 school children. The clinical group consisted of 90 children with prematurely extracted teeth. The patients from the clinical group were divided into three groups of 30 patients. The control group consisted of 50 children with intact denture.

* Patients who have lost one or more tooth through extraction, who have come to the clinic during the first 2 month after the extraction and have no orthodontic problems.

* Patients with prematurely extracted temporary teeth, 6 or more months prior, with mild orthodontic problems.

* Patients with premature temporary tooth loss, 6 or more months prior, who had severe orth- amined children are too young (6-9 years old) and have no calculus. Criteria for classifying the amount of plaque:

\section{0 - No plaque or stain present.}

1 - Plaque covering not more than one third of the tooth surface, or presence of extrinsic stains without other debris regardless of the surface area covered.

2 - Plaque covering more than one third, but not more than two thirds, of the exposed tooth surface.

3 - Plaque covering more than two thirds of the exposed tooth surface.

The index is calculated as an average sum of the estimates of the explored surfaces and their number.

The index is based on clinical observation of the stained teeth of patients with dental mirror, cotton pellets and suction $(2,5)$.

\section{RESULTS AND DISCUSSION}

The differences in the OHI, between the control group and the clinical groups are presented in Table 1.

Table 1. Comparison of the OHI index, between the control group and the clinical group

\begin{tabular}{lc|c|c|cc} 
& control $(\mathrm{n}=50)$ & Group 1 $(\mathrm{n}=30)$ & Group 2 $(\mathrm{n}=30)$ & Group 3 $(\mathrm{n}=30)$ & \\
& mean \pm SEM & mean \pm SEM & mean \pm SEM & mean \pm SEM & \\
index & $1.86 \pm 0.05$ & $1.78 \pm 0.06$ & $1.90 \pm 0.08$ & $2.01 \pm 0.05$ & 0,01 \\
\hline OHI Greene Vermillion & & &
\end{tabular}

odontic problems (II and III class Angle).

* The control group consisted of 50 children of the same age, who had no premature extraction.

The oral hygiene status is examined and registered according to the WHO by the Greene-Vermillion OHI-S. This index is one of the most often used in the epidemiology to estimate the distribution of plaque, by coloring the teeth. The OHI-S, like the OHI, has two components, the Plaque Index and the Calculus Index. Each of these indexes, in turn, is based on numerical determinations representing the amount of plaque or calculus found on the preselected tooth: the vestibular surfaces of the teeth $(1,3,5,8)$ and the lingual surfaces of the teeth $(4,6)$. We haven't used the Calculus Index in our study, because the ex-
There is a statistically significant difference in the $\mathrm{OHI}-\mathrm{S}$ of the control group and the clinical groups as well as between the clinical groups.

The children of the control group (without prematurely extracted teeth) and the first clinical group have the lowest OHI $-S 1.86 \pm 0.05$ and $1.78 \pm 0.06$, respectively. The results are shown in a graph by Boxplot diagram on Fig. 1.

On the Box-plot diagram the statistically significant differences between the OHI Green Vermillion of the controlled and the clinical groups are seen. There are some cases from the control group, the second and the third group with an extremely high OHI-S index of more than 2.5. The values of the OHI-S Green Vermillion of the control and the 


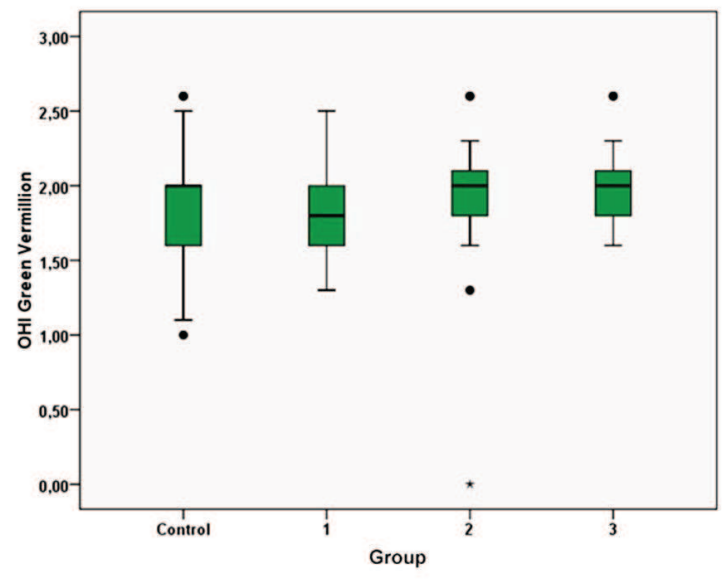

Fig. 1. Box-plot diagram for the OHI-S index in the control group and the clinical groups

clinical groups are relatively close. The third clinical group has the highest average values of the OHI-S index (2.01). It means that they have the worst oral hygiene. The explanation of this result is that the patients with malocclusions could hardly have proper oral hygiene, because of the many retentive for the plaque places on their teeth. The poor oral hygiene of these children could also be because of the pain, when they are brushing their teeth, as a result of their neglected oral health. The OHI-S index for the second group is 1.90. The children from the control and the first clinical groups have lower OHI-S of Greene Vermillion (1.86 and 1.78) than the other two groups, because of their better oral hygiene.

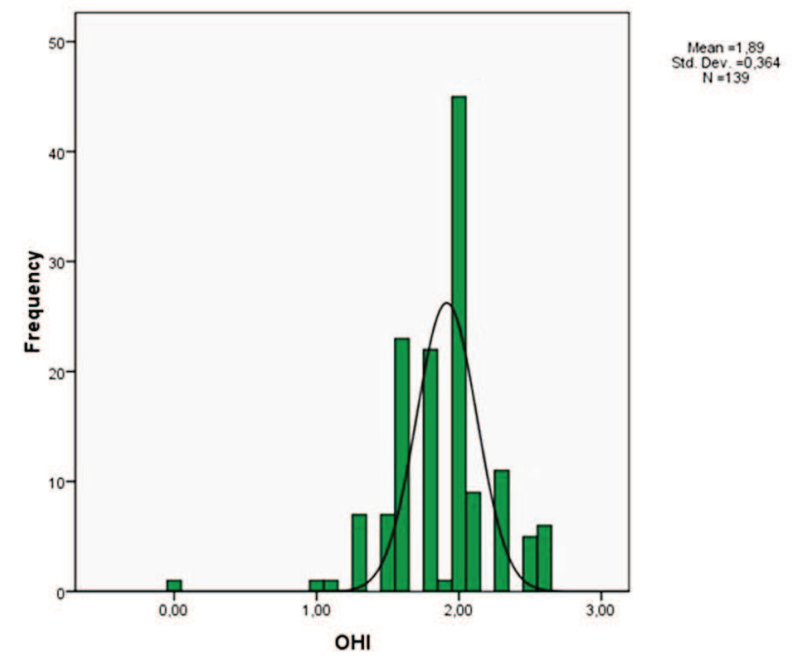

Fig. 2. Histogram of the OHI-S
This result confirms that the relationship between the oral hygiene, caries activity and the orthodontic deformation is in both directions. Children with orthodontic deformation have higher plaque retention and a higher risk of caries as a result. The caries complication also leads to premature tooth extraction which is one of the risk factors for the development of orthodontic deformation. Our results are confirmed by Ribagin and other autors $(6,7,9,10)$. The results of the statistical analysis show the uneven distribution of the OHI-S index (Fig. 2).

There are children with very high and very low OHI-S indexes - under 1.00 and above 3.00, which differ significantly from the average values - about 2.00 That is why for the intergroup analyses we have used Kruskal Wallis's method.

\section{CONCLUSION}

The data from the oral hygiene status show that children with premature extraction of the temporary teeth have poor oral hygiene compared to control group (children without early loss of teeth). The children from the third group, with prematurely extracted temporary teeth and severe orthodontic deformation have the worst oral hygiene. This results demonstrate the connection between the oral hygiene, the malocclusions and the need of prophylaxis.

\section{REFERENCES}

1. Edward Lo. Caries prevention Strategies. Epidemiology. Chicago: Quintessence. 2005;10(4):19-23.

2. Drury T, Horowitz A. Diagnozing and reporting early chaild caries for research purpose. J.Publ. Health Dent.1999;59:192-197.

3. Kelner N, Rodrigues MJ, Miranda K. Prevelance of early loss of desiduous molars in children attending the FOP/UPE in 2002 and 2003. Dent Clin Sci Recife. 2005;4:213-8.

4. Greenberg R. Madical epidemiology. Prentice Hall International Inc. 1995;146.

5. Greene JC, Vermillion JR. The simplified oral hygiene index. Journal of American Dental Association. 1964;68:25-31.

6. Gulsum AK, Elif Sepet, Arzu Pinar,Gamze Aren, Oral Health Prev. Dent 2005; 3:113-117.

7. Melsen B, Terp S. 1982. The influence of extractions caries causa on the developmet of maloc- 
clusion and need for orthodontic treatment. Swed Dent J Supplement. 1982;15:163-169.

8. Northway WM, Wainright RW. D E space--a realistic measure of changes in arch morphology: space loss due to unattended caries. J Dent Res. 1980;59:1577-80.

9. Peneva M. Response rates of tooth decay. In:Caries in the $21^{\text {st }}$ century. East-West. 2008;29-33. Bulgarian.

10. Ribagin L. Changes in oral status in children with tooth-jaw deformities. (dissertation). Sofia.2015;5-47. Bulgarian.

11. Vargas C, Ronzio S. Disparities in Early Chaildhood Caries. BMC Oral Health. 2006;6-23. 